'Departamento de

Gastroenterología,

Facultad de Medicina,

Pontificia Universidad Católica de

Chile. Santiago, Chile.

Este artículo de revisión no recibió financiamiento.

El autor declara no tener conflictos de interés.

Recibido el 8 de enero de 2019 aceptado el 29 de enero de 2020.

Correspondencia a: Dr. Roberto Candia MSc.

Departamento de

Gastroenterología

Facultad de Medicina Pontificia Universidad Católica de Chile.

Edificio Académico Facultad de Medicina UC

Diagonal Paraguay 362 4to piso, oficina 424 Santiago, Chile. roberto.candia@gmail.com

\section{Herramientas para la interpretación de informes endoscópicos: clasificaciones y escalas en endoscopía digestiva}

\author{
ROBERTO CANDIA ${ }^{1}$
}

\section{Classifications and scores used in digestive endoscopy}

\begin{abstract}
Interpretation and description of findings detected in upper-endoscopy and colonoscopy are qualitative processes which depend on the experience and skills of the endoscopist performing the procedure. This explains the high variability of endoscopic reports, hampering their interpretation, specially by general practitioners. Classifications, scores and scales give a quantitative support to these qualitative processes. The aim of this review is to describe the classifications, scores and scales most frequently reported in digestive endoscopy, specially those with the highest methodological support in terms of validation and reproducibility. These tools facilitate the description of findings related to gastroesophageal reflux, Barrett's esophagus, gastroesophageal varices, stigmas related to non-variceal gastrointestinal bleeding, advanced and incipient neoplasms, bowel preparation for colonoscopy and severity scores of inflammatory bowel diseases. In summary, these tools enable to standardize endoscopic reports, simplifying their interpretation.
\end{abstract}

(Rev Med Chile 2020; 148: 992-1003)

Key words: Esophageal and Gastric Varices; Gastrointestinal Hemorrhage; Inflammatory Bowel Diseases; Gastrointestinal Neoplasms; Gastroesophageal Reflux.

\section{L} a endoscopía digestiva alta y la colonoscopia son exámenes operador dependiente. La interpretación y descripción de los hallazgos son procesos cualitativos que dependen de la experiencia y conocimiento del endoscopista que informa el procedimiento. En nuestro medio no existen normas que estandaricen la realización de los reportes endoscópicos, lo que determina una amplia variabilidad en la calidad de los informes, lo que dificulta su interpretación. La solución es una descripción estandarizada de los hallazgos. Para ello se han creado clasificaciones y escalas, las que buscan dar consistencia objetiva a las apreciaciones subjetivas de los endoscopistas. Si bien estas clasificaciones facilitan la descripción e interpretación de los exámenes endoscópicos, su divulgación en nuestro medio aún es limitada. Los objetivos de esta revisión son describir las clasificaciones más utilizadas en endoscopía digestiva tradicional, es decir, endoscopia con luz blanca sin magnificación ni tinción, y detallar el respaldo metodológico que las sustenta, en un esfuerzo de facilitar la interpretación de los informes endoscópicos tanto para médicos especialistas como no especialistas.

\section{Respaldo científico de las clasificaciones endoscópicas}

El proceso científico para dar consistencia cuantitativa a hallazgos cualitativos es complejo. Desde el punto de vista metodológico, este proceso es llamado validación. Los estudios de validación 
se dividen en varias etapas ${ }^{1}$, las que se describirán someramente a continuación. Uno de los aspectos más relevantes es que la escala de alguna forma cuantifique la relevancia clínica de los hallazgos, es decir, debe medir apropiadamente el constructo teórico que dice medir. Una forma de validar este punto es comparar la clasificación endoscópica con otra herramienta que mida el mismo concepto o constructo, una especie de estándar de oro. A modo de ejemplo, las escalas que evalúan las várices esofágicas (VE) buscan medir en forma simple el gradiente de presión porto-sistémico y el riesgo de hemorragia. Una buena clasificación se debe correlacionar bien con ambos constructos. Otro aspecto es la validación de contenido, en donde la escala debe reflejar todos los aspectos importantes de lo que se quiere medir. Por ejemplo, las escalas de gravedad endoscópica de enfermedad inflamatoria intestinal (EII) deben considerar las características que mejor reflejan la inflamación de la mucosa: eritema, friabilidad, erosiones, úlceras. Una buena clasificación también debe tener consistencia interna, es decir, debe variar si la condición varía. Por ejemplo, una buena escala de gravedad endoscópica de EII debe mejorar o empeorar en concordancia con la mejoría o deterioro del paciente. Finalmente, la escala debe ser reproducible, es decir, debe existir concordancia interobservador (los mismos hallazgos reciben la misma clasificación por 2 operadores) e intraobservador (el mismo observador evalúa los hallazgos de la misma forma en distintas circunstancias) $)^{2,3}$.

A continuación, se describirán brevemente las clasificaciones endoscópicas de mayor uso junto a su respaldo metodológico.

\section{Clasificación de Los Ángeles}

El reflujo gastroesofágico (RGE) es una condición frecuente, pero menos de $40 \%$ de los pacientes con RGE tienen lesiones reconocibles en la endoscopia ${ }^{4-6}$. Las erosiones del esófago distal son el hallazgo endoscópico de mayor correlación con RGE y la clasificación de Los Ángeles ${ }^{7}$ permite etapificar la esofagitis erosiva en 4 niveles de gravedad (Figura 1). Publicada en 1996, es una clasificación simple, tiene buena correlación con la pHmetría esofágica de 24 h (estándar de oro para el diagnóstico de RGE), alta concordancia interobservador (kappa $=0,8-0,9)$ y ha sido validada en varias poblaciones ${ }^{6,8,9}$. A pesar de lo anterior, los consensos modernos consideran que solo las esofagitis erosivas grado C o D de Los Ángeles, junto con el esófago de Barrett (EB) y la estenosis péptica de la unión gastroesofágica (UGE), son hallazgos endoscópicos que por sí solos permiten hacer el diagnóstico de RGE patológico ${ }^{10}$. La esofagitis grado A se considera un hallazgo inespecífico, ya que puede ser encontrada en hasta $7,5 \%$ de controles sanos. La esofagitis grado B correctamente evaluada se correlaciona bien con RGE patológico, pero problemas de variabilidad interobservador limitan su utilidad como elemento diagnóstico único ${ }^{10}$. En suma, esta es una clasificación útil que debe ser utilizada en la práctica clínica, pero no es perfecta.

Otros hallazgos frecuentemente descritos en relación a RGE, como cambios mínimos no erosivos de la mucosa esofágica (eritema, congestión, hiperqueratosis) o la clasificación de Savary-Miller, descrita en $1968^{11}$, no han sido validados y no es clara su correlación con la pHmetría esofágica, por lo que no debiesen ser utilizados en la práctica habitual.

\section{Clasificación de Hill}

Otra escala relacionada a RGE es la clasificación de Hill. Esta clasificación evalúa la UGE posicionando el endoscopio en el cuerpo gástrico para observan en retrovisión el esfínter esofágico inferior. En una UGE normal el esfínter comprime y se adhiere completamente a la circunferencia del endoscopio (UGE tipo I según Hill). Una UGE laxa, dada por la formación de un espacio entre el esfínter y el endoscopio, se considera anormal (tipo II y III). En casos extremos, como en una hernia hiatal, el diámetro del esfínter es superior al del endoscopio, dejando un espacio permanente entre ambas estructuras (tipo IV) ${ }^{12,13}$. A pesar de su amplia difusión, su capacidad para predecir RGE patológico es limitada. Por esta razón, los consensos modernos no la consideran como una herramienta diagnóstica válida ${ }^{10}$. Su utilidad es meramente descriptiva.

\section{Clasificación de Praga}

El EB se define como la aparición de metaplasia intestinal en el epitelio escamoso del esófago distal. Endoscópicamente se manifiesta como el 


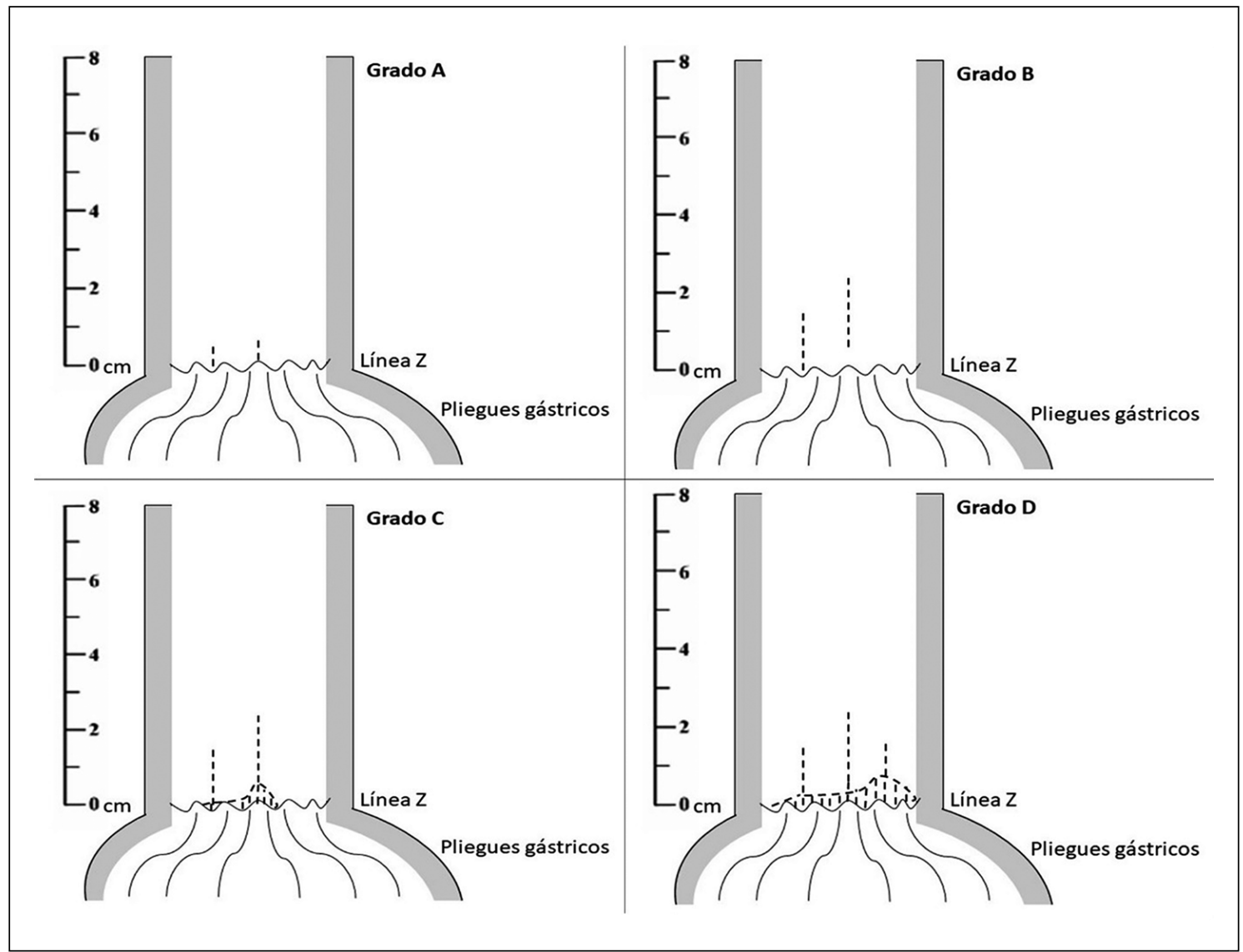

Figura 1. Clasificación de Los Ángeles. Esta clasificación divide la esofagitis erosiva en 4 etapas según su severidad: Grado A: Una o varias erosiones limitadas a los pliegues mucosos (longitudinales) y $<5 \mathrm{~mm}$ de extensión. Grado B: Una o varias erosiones limitadas a los pliegues mucosos y > $5 \mathrm{~mm}$ de extensión. Grado C: Erosiones que se extienden lateralmente sobre los pliegues mucosos longitudinales, pero que compromete menos del 75\% de la circunferencia. Grado D: Erosiones longitudinales y con extensión lateral confluentes, que se extienden más del 75\% de la circunferencia ${ }^{7,8}$. Las erosiones están representadas por líneas punteadas.

ascenso de la línea $\mathrm{Z}$ o la aparición de lengüetas de mucosa gástrica en el esófago distal. Su importancia radica en que es una lesión precursora de adenocarcinoma esofágico. La clasificación de Praga fue creada en 2006 para estandarizar la extensión del $\mathrm{EB}^{14}$. Consiste en medir la longitud del EB mediante 2 parámetros, $\mathrm{C}$ y $\mathrm{M}$. El parámetro $\mathrm{C}$ corresponde a la longitud del segmento circunferencial, el parámetro $\mathrm{M}$ corresponde a la longitud máxima del EB, dado por la lengüeta más larga (Figura 2). Su importancia radica en que, a mayor longitud, mayor riesgo de adenocarcinoma. Esta clasificación es simple, tiene alta concordancia interobservador (coeficiente de correlación intraclase $(\mathrm{CCI})>0,9)$ y está validada en población occidental y oriental ${ }^{15-17}$, por lo que debe ser utilizada. Antiguamente el EB se describía como largo $(>3 \mathrm{~cm})$, corto $(<3 \mathrm{~cm})$ y ultracorto $(<1 \mathrm{~cm})^{18}$. Esta es una clasificación obsoleta y no debiese ser utilizada. Adicionalmente, la evidencia actual establece que las lengüetas $<1 \mathrm{~cm}$ (EB ultracorto) no tienen relevancia clínica, por lo que no debiesen ser informadas como $\mathrm{EB}^{19}$.

\section{Várices esofágicas}

Múltiples son las clasificaciones disponibles. La que usamos actualmente deriva de 3 clasificaciones 


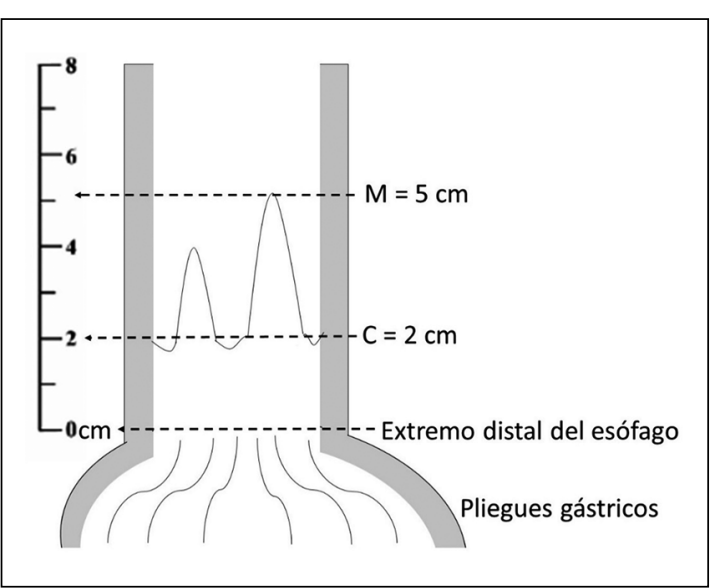

Figura 2. Clasificación de Praga para la extensión del esófago de Barrett (EB). El parámetro $C$ y el parámetro $M$ se miden desde el límite inferior del esófago, definido por el inicio de los pliegues gástricos. El parámetro $\mathrm{C}$ se mide hasta la extensión circunferencial de la metaplasia, el parámetro $\mathrm{M}$ hasta la máxima extensión de la metaplasia (lengüeta más larga). Dado que ambos parámetros se miden desde el extremo distal del esófago, nunca el parámetro $C$ es mayor que el M. Este esquema representa un EB C2M5 de la clasificación de Praga ${ }^{14}$.

desarrolladas hace más de 30 años. La clasificación de Paquet ${ }^{20}$ fue propuesta en un estudio aleatorizado de escleroterapia, clasificando las várices en 3 grados según su tamaño:

- Grado I: Varices que se extienden mínimamente sobre la mucosa (desaparecen con la insuflación).

- Grado II: Várices proyectándose hasta 1/3 del lumen (no se comprimen con la insuflación).

- Grado III: Várices proyectándose hasta 50\% del lumen y/o se contactan unas con otras.

La sociedad japonesa de estudio de la hipertensión portal ${ }^{21}$ estableció una descripción de las várices basado en 4 aspectos:

- Color: Blanco o azul.

- Signos rojos: Ausentes o presentes.

- Forma: Rectas, tortuosas o muy largas y tortuosas.

- Localización: Tercio distal, medio o superior.

Ambas clasificaciones están consideradas en la escala propuesta por el Club Endoscópico del Norte de Italia ${ }^{22}$. En esta escala, las várices se describen en base a 3 aspectos:
- Clasificación Child-Pugh: A, B, C.

- Tamaño de las várices: Pequeñas o grandes. Estas últimas corresponden a las várices medianas y grandes de la clasificación de Paquet. - Signos rojos: Ausentes o presentes, igual que la clasificación japonesa.

Todas estas clasificaciones son subjetivas, tienen escasos estudios de validación y, en al menos un estudio, la concordancia interobservador fue baja (kappa entre 0,3 y 0,6$)^{23}$. A pesar de lo anterior, la clasificación italiana fue validada en base a una cohorte prospectiva. Este estudio incluyó 321 pacientes cirróticos y se evaluó el riesgo del primer episodio de hemorragia a 2 años de seguimiento (Figura 3). Dada su capacidad para predecir pronóstico, es la clasificación más utilizada ${ }^{22}$. Su utilidad radica en que permite seleccionar los pacientes que requieren profilaxis primaria de hemorragia variceal $^{24}$ (Figura 3 ).

\section{Várices gástricas}

La clasificación de Sarin fue propuesta en el año 1989 y divide las várices gástricas (VG) según su localización y su relación con las $\mathrm{VE}^{25}$. De acuerdo a Sarin, existen 2 tipos de VG, las várices gastroesofágicas (VGE) y las várices gástricas aisladas (VGA), las que a su vez se dividen en tipo 1 y 2 (Figura 4). Epidemiológicamente, las VGE tipo 1 son las más frecuentes y representan $75 \%$ de todas las VG, mientras que las VGE tipo 2, las VGA tipo 1 y las VGA tipo 2 representan 15\%, 8\% y $2 \%$, respectivamente $e^{25,26}$. La principal utilidad de esta clasificación es que guía la terapia endoscópico de las VG. En los pacientes con VGE tipo 1 la ligadura con bandas elásticas es la primera opción, en cambio, en las VGE tipo 2 y las VGA la primera opción es la escleroterapia con cianoacrilato ${ }^{24,26}$. A pesar de lo anterior, el grado de concordancia interobservador para esta clasificación no está documentada.

\section{Clasificación de Forrest}

Esta clasificación fue publicada en $1974^{27}$ con el objetivo de etapificar el riesgo de resangrado de lesiones ulceradas en cualquier porción del tubo digestivo (Tabla 1). A pesar de los avances terapéu- 


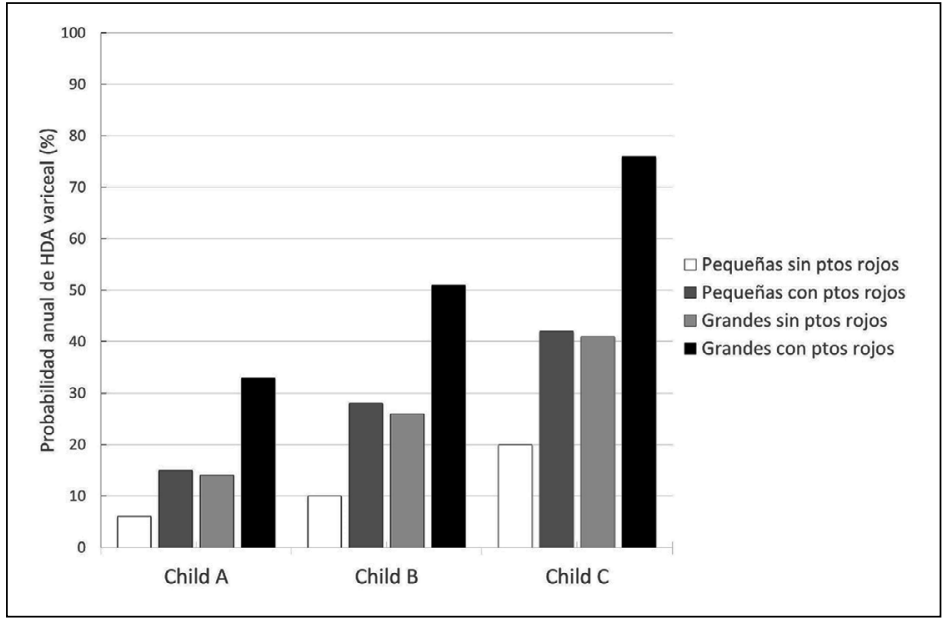

Figura 3. Riesgo de padecer el primer episodio de hemorragia digestiva variceal en cirróticos según clasificación Child-Pugh y la descripción endoscópica de las varices (tamaño y presencia de puntos rojos). Todos los pacientes con riesgo mayor a $10 \%$ anual son de alto riesgo y se debe considerar iniciar profilaxis primaria para hemorragia variceal. En términos simples, todos los pacientes Child-Pugh $\mathrm{C}$, independiente de la morfología de las várices, y todos los pacientes con várices grandes $\mathrm{y} / \mathrm{o}$ con puntos rojos, independiente del Child, deben ser considerados de alto riesgo ${ }^{22,24}$.

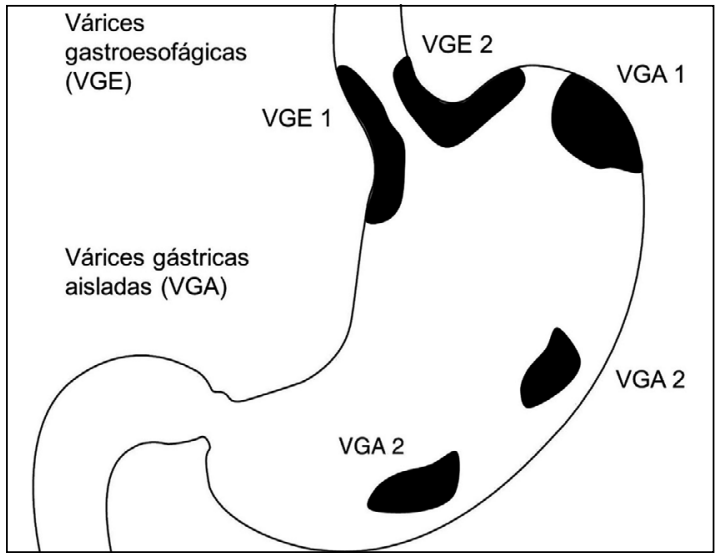

Figura 4. Clasificación de Sarín para várices gástricas (VG). Esta clasificación divide las VG en 2 tipos, várices gastroesofágicas (VGE) y várices gástricas aisladas (VGA). Las VGE son aquellas várices que nacen en el esófago y se extienden al estómago. Aquellas que se extienden por la curvatura menor son llamadas VGE tipo 1. Las VGE que se extienden por la curvatura mayor hacia el fondo gástrico son llamadas VGE tipo 2. Por otro lado, las VGA también se dividen en 2 tipos. Las VGA tipo 1 son aquellas ubicadas en el fondo, pero no pasan al esófago ni alcanzan el cardias. Las VGA tipo 2 son aquellas várices ectópicas ubicadas en cualquier otra porción del estómago ${ }^{24-26}$.

ticos, como la incorporación de los inhibidores de bomba de protones (IBP) y la mejoría tecnológica en hemostasia, esta clasificación sigue vigente y fue recientemente revalidada mediante una cohorte prospectiva $^{28}$. De acuerdo a este estudio, el riesgo de resangrado a 30 días es alto para las lesiones tipo Ia, Ib, IIa y IIb, (59\%, 26\%, 21\% y 31\%, respectivamente), por lo que la recomendación es realizar terapia endoscópica y usar IBP en dosis altas. Para las lesiones tipo IIb, los datos que apoyan la terapia endoscópica son menos categóricos y se debe decidir caso a caso ${ }^{29}$. En cambio, las lesiones IIc y III se consideran de bajo riesgo de resangrado (15\% y $6 \%$ a 30 días, respectivamente), no requerirían terapia endoscópica y pueden ser manejadas solo con IBP ${ }^{28,29}$.

\section{Clasificación de Borrmann}

Esta clasificación fue descrita por anatomopatólogos en 1926, varias décadas antes de la invención del endoscopio. Actualmente se utiliza para describir el cáncer gástrico avanzado, sin embargo, es una clasificación no validada, imprecisa y con mala correlación pronóstica. Divide las neoplasias gástricas avanzadas en 5 tipos $^{30,31}$ :

- Tipo 1: Polipoídea.

- Tipo 2: Ulcerada con bordes netos definidos.

- Tipo 3: Ulcerada con bordes mal definidos (infiltrativo).

- Tipo 4: Infiltrativo, predominantemente intramural, pobremente demarcado.

- Tipo 5: No clasificable.

La asociación japonesa de cáncer gástrico publicó su propia clasificación en $1998^{30}$. Esta 
Tabla 1. Clasificación de Forrest para la etapificación del riesgo de sangrado de lesiones ulceradas del tubo digestivo

\begin{tabular}{|ll|}
\hline Forrest I: Sangrado activo & $\begin{array}{l}\text { la: Sangrado en jet (arterial) } \\
\text { Ib Sangrado en napa (venoso) }\end{array}$ \\
Forrest II: Estigmas de sangrado reciente & Ila: Vaso visible (coágulo plaquetario $<5 \mathrm{~mm}$ ) \\
& $\begin{array}{l}\text { Ilb: Coágulo adherido (coágulo }>5 \mathrm{~mm} \text { ) } \\
\text { Ilc: Úlcera de fondo sucio. }\end{array}$ \\
Forrest III: Sin estigmas de sangrado reciente & III: Úlcera de fondo limpio \\
\hline
\end{tabular}

iniciativa incluye la clasificación de Borrmann y agrega el estadío "0" para describir neoplasias no avanzadas, es decir, aquellas que se extienden solo hasta la submucosa. Esta iniciativa fue incorporada al mundo occidental con el nombre de Clasificación de Paris.

\section{Clasificación de Paris}

Esta clasificación fue propuesta en el año 2002 y tiene como objetivo caracterizar las lesiones neoplásicas de todo el tubo digestivo. Divide las lesiones en 6 tipos, desde el tipo 0 al 5. Los estadíos 1 al 5 corresponden a neoplasias avanzadas según Borrman, es decir, aquellas que alcanzan la capa muscular propia. El estadío 0 corresponde a lesiones incipientes, es decir, aquellas que pueden ser resecadas endoscópicamente con opción curativa. El estadío 0, a su vez, se divide en 3 subtipos: pólipos (0-I), lesiones superficiales o planas (0-II) y lesiones excavadas (0-III) (Figura 5) ${ }^{31}$.

Esta clasificación es relevante porque permitió establecer y difundir que las neoplasias del tracto digestivo alto, especialmente el adenocarcinoma gástrico y los carcinomas esofágicos, provienen de lesiones planas difíciles de detectar endoscópicamente (tipo 0-II o 0-III), a diferencia del adenocarcinoma colorrectal, que proviene de lesiones polipoídeas (tipo 0-I). A pesar de lo anterior, esta clasificación no está totalmente validada y está basada predominantemente en la experiencia

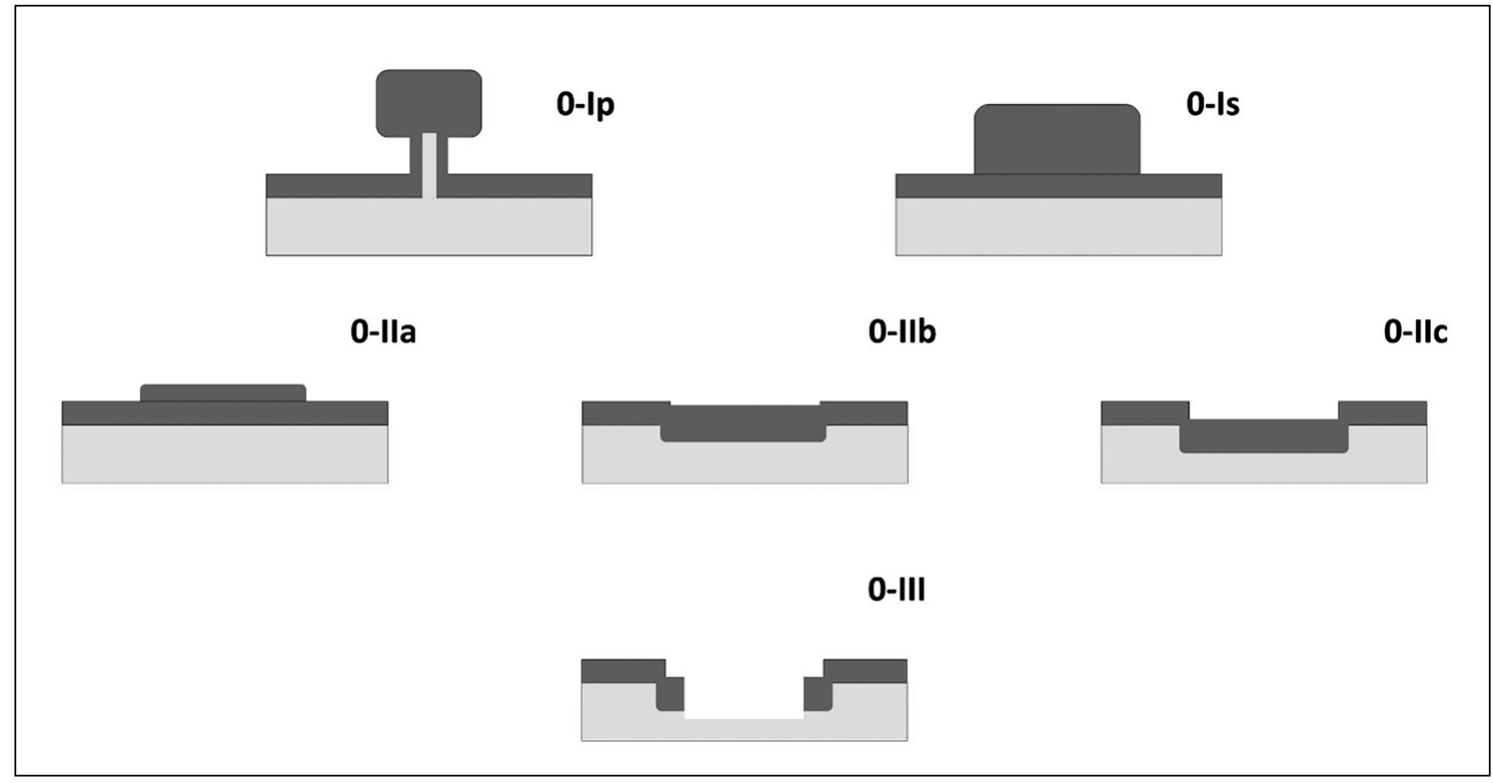

Figura 5. Representación esquemática de las lesiones tipo 0 o incipientes de la clasificación de París. 0-Ip: Pólipo pediculado; 0-Is: Pólipo sésil; 0-Ila: Lesión superficial elevada; 0-IIb: Lesión superficial plana; OII-c: Lesión superficial deprimida; 0-III: Lesión excavada (úlcera superficial) ${ }^{31}$. El color gris oscuro representa a la mucosa, el color gris claro la submucosa. 
oriental. Un estudio reciente intentó validarla entre endoscopistas occidentales expertos, logrando una concordancia interobservador solo moderada $($ kappa $=0,38)$, por lo que su reproducibilidad ha sido cuestionada ${ }^{32}$. A pesar de estos datos, es una clasificación relevante, ya que ha permitido aumentar la detección de neoplasias gástricas en etapa incipiente, mejorando su probabilidad de curación ${ }^{31}$.

\section{Clasificación de Boston}

Esta clasificación fue publicada en 2009 y su objetivo es calificar la calidad de la limpieza en una colonoscopía ${ }^{33}$. La preparación del colon es fundamental, ya que la capacidad para detectar lesiones se correlaciona directamente con el grado de limpieza de la mucosa ${ }^{33}$. Esta escala divide la calidad de la preparación en 4 niveles (Tabla 2). Una preparación es óptima cuando todos los segmentos son evaluados con 3 puntos ( 9 puntos totales). Un puntaje $\geq 6$ (con al menos 2 puntos en cada segmento) se considera una preparación adecuada $^{34}$. Colonoscopias con un puntaje menor son insuficientes como examen de tamizaje para adenomas y cáncer colorrectal.

Esta escala tiene alta concordancia interobservador $(\mathrm{CCI} \geq 0,74)$, ha sido validada en diferentes poblaciones y se correlaciona con eventos clínicos relevantes (detección de pólipos), por lo que debe ser utilizada ${ }^{33-35}$.

\section{Clasificaciones en EII}

El diagnóstico y la evaluación de gravedad de la colitis ulcerosa (CU) y enfermedad de Crohn (EC) dependen en gran parte de los hallazgos endoscópicos. Ambas condiciones se presentan con mayor gravedad mientras mayor es la extensión de la enfermedad y mayor es el grado de inflamación del segmento comprometido ${ }^{36,37}$. En la actualidad, la curación de la mucosa es un objetivo terapéutico deseable tanto para CU como EC. La evidencia disponible muestra que el pronóstico a largo plazo es mejor en pacientes asintomáticos con mucosa endoscópicamente sana que en aquellos con inflamación persistente, tanto en términos de recaídas y hospitalizaciones como necesidad de cirugía ${ }^{38-41}$. La magnitud de la inflamación se manifiesta por eritema, friabilidad, sangrado espontáneo, erosiones y úlceras. En colon, la disminución de la transparencia mucosa, manifestada por pérdida de la visualización de vasos submucosos, también es un signo de inflamación. Adicionalmente, para la EC se agrega la presencia de estenosis. Todos estos elementos han sido considerados para construir múltiples escalas de gravedad (Tabla 3 ).

Para CU, la escala más utilizada es el Mayo Clinic subscore endoscópico. Originalmente fue diseñada para evaluar la gravedad de la enfermedad en un estudio aleatorizado del año $1987^{42}$. La escala original incluye parámetros clínicos más la evaluación endoscópica. El subscore endoscópico tiene 4 niveles (Tabla 4). Esta escala ha sido ampliamente utilizada en estudios clínicos y está considerada en la legislación chilena para financiar la terapia biológica ${ }^{43}$, pero no está validada ${ }^{44}$. Su principal ventaja es su simpleza, sin embargo, no incluye en su definición la extensión de la enfermedad, las definiciones para cada estrato son laxas y su consistencia interna es baja. Nuevas escalas han intentado soslayar estas limitaciones. A modo de ejemplo, UCEIS (Ulcerative Colitis Endoscopic Index of Severity) es una escala recientemente desarrollada que podría ser el estándar en el corto plazo. Es una escala simple, metodológicamente bien diseñada, está validada y tiene buena concordancia interobservador ${ }^{44-47}$ (Tabla 5).

\section{Tabla 2. Clasificación de Boston para evaluar la limpieza del colon durante una colonoscopia}

\begin{tabular}{|ll}
\hline Boston 0 & No preparado, mucosa no visualizada por deposición sólida que no puede ser lavada \\
Boston 1 & Mucosa parcialmente visualizada, el resto oculta por deposición sólida residual o líquido opaco \\
Boston 2 & Cantidad menor de deposición residual o líquido opaco, la mucosa se logra evaluar satisfactoriamente \\
Boston 3 & Mucosa totalmente visualizada, sin deposición ni líquido opaco
\end{tabular}

El cálculo final de esta escala implica sumar la calidad de la preparación en 3 segmentos, colon derecho, colon transverso y colon izquierdo-sigmoides, por lo tanto, el puntaje varía entre 0 a 9 puntos. A mayor puntaje, mejor preparación del colon³. 
Tabla 3. Algunos ejemplos de escalas endoscópicas de gravedad descritas para colitis ulcerosa y enfermedad de Crohn

\begin{tabular}{|ll|}
\hline Colitis ulcerosa & Enfermedad de Crohn \\
\hline Baron score & CDEIS \\
\hline Blackstone Endoscopic interpretation & SES-CD \\
\hline Matts index & Rutgeert's score \\
\hline Mayo clinic endoscopy subscore & \\
\hline Modified Baron endoscopy score & \\
\hline Modified Mayo Clinic subscore & \\
\hline UCCIS & \\
\hline UCDAI & \\
\hline UCEIS & \\
\hline
\end{tabular}

Las de mayor relevancia están descritas en el texto. UCCIS: Ulcerative Colitis Colonoscopic Index of Severity; UCDAI: Ulcerative Colitis Disease Activity Index; UCEIS: Ulcerative Colitis Endoscopic Index of Severity; CDEIS: Crohn's Disease Endoscopic Index of Severity; SES-CD: Simple Endoscopic Score for Crohn's Disease.

Tabla 4. Clasificación endoscópica de Mayo para la gravedad de la colitis ulcerosa

\begin{tabular}{|ll|}
\hline Mayo 0 & Mucosa normal \\
\hline Mayo 1 & Eritema y granularidad leve, leve disminución de patrón vascular submucoso \\
Mayo 2 & Eritema franco, friabilidad, sangramiento al contacto, patrón vascular ausente \\
\hline Mayo 3 & Úlceras y sangrado espontáneo \\
\hline
\end{tabular}

Se informa el puntaje del segmento más comprometido. La curación endoscópica de la mucosa se alcanza cuando la mucosa es Mayo 0. En algunos estudios el Mayo 1 también es considerado remisión endoscópica ${ }^{42,44}$.

Para la EC, la primera gran iniciativa fue el CDEIS (Crohn's disease endoscopic index of severity $)^{48}$. Esta escala divide la colonoscopia en 5 segmentos (íleon, colon derecho, transverso, izquierdo-sigmoides y recto) y para cada uno evalúa la presencia de úlceras (profundas y superficiales), mide la extensión tanto de las úlceras como de la inflamación y la presencia de estenosis, todo corregido por el número de segmentos examinados (Figura 6A). Si bien esta escala ha sido validada por otros grupos, es muy demandante en términos de tiempo, por lo que es poco utilizada en la práctica habitual. El mismo grupo desarrolló una versión simplificada (SES-CD, Simple Endoscopic Score for Crohn's Disease ${ }^{49}$, en donde cada parámetro (tipo y extensión de las úlceras, extensión de la inflamación y presencia de estenosis) es categorizado en 3 niveles y se suma el puntaje de los hallazgos de los 5 segmentos antes descritos
(Figura 6B). Esta nueva versión ha sido validada en varias poblaciones, tiene alta concordancia interobservador (kappa $>0,8)$ y alta correlación con CDEIS ${ }^{50}$. Finalmente, otra escala importante es el Índice endoscópico de Rutgeerts. Esta escala permite diagnosticar la reactivación endoscópica de EC después de una resección quirúrgica. Se mide a nivel de la anastomosis y evalúa la presencia de aftas, eritema, úlceras y estenosis. Los hallazgos se dividen en 5 estratos, llamados i0 a i4, siendo i0 mucosa normal e i4 úlceras profundas y/o estenosis (Tabla 6). Si bien no ha sido formalmente validada, ha sido utilizada en múltiples estudios para guiar la terapia en contexto de reactivación endoscópica de la EC (puntaje $\geq \mathrm{i} 2$ ) $^{51,52}$. En Chile es un parámetro considerado en la legislación para financiar la terapia biológica posrresección quirúrgica (puntaje $\geq i 3$ ), por lo que debe ser utilizada en la práctica habitual ${ }^{53}$. 
Tabla 5. Puntajes y definiciones para la escala UCEIS (Ulcerative Colitis Endoscopic Index of Severity)

\begin{tabular}{|c|c|c|}
\hline Descriptor & Puntaje & Definición \\
\hline \multirow[t]{3}{*}{ Patrón vascular } & $\begin{array}{l}\text { Normal } \\
0 \text { puntos }\end{array}$ & $\begin{array}{l}\text { Patrón vascular normal con arborización de capilares cuyos bordes } \\
\text { están claramente definidos o levemente borrosos }\end{array}$ \\
\hline & $\begin{array}{l}\text { Pérdida del patrón en parches } \\
1 \text { punto }\end{array}$ & Pérdida irregular o en parches de la visualización del patrón vascular \\
\hline & $\begin{array}{l}\text { Pérdida completa del patrón } \\
2 \text { puntos }\end{array}$ & Pérdida completa de la visualización del patrón vascular \\
\hline \multirow[t]{4}{*}{ Sangrado } & $\begin{array}{l}\text { Sin sangrado } \\
0 \text { puntos }\end{array}$ & No hay sangre visible \\
\hline & $\begin{array}{l}\text { Mucoso } \\
1 \text { puntos }\end{array}$ & $\begin{array}{l}\text { Algunos puntos o líneas de sangre coagulada sobre la mucosa, la que } \\
\text { puede ser lavada }\end{array}$ \\
\hline & $\begin{array}{l}\text { Luminal leve } \\
2 \text { puntos }\end{array}$ & Escasa sangre líquida libre en el lumen \\
\hline & $\begin{array}{l}\text { Luminal moderado o grave } \\
3 \text { puntos }\end{array}$ & $\begin{array}{l}\text { Sangre líquida franca en el lumen o sangrado espontáneo desde una } \\
\text { mucosa hemorrágica después de lavar el lumen }\end{array}$ \\
\hline \multirow[t]{4}{*}{$\begin{array}{l}\text { Erosiones y } \\
\text { úlceras }\end{array}$} & $\begin{array}{l}\text { Sin lesiones } \\
0 \text { puntos }\end{array}$ & Mucosa normal \\
\hline & $\begin{array}{l}\text { Erosiones } \\
1 \text { punto }\end{array}$ & $\begin{array}{l}\text { Pequeños defectos de continuidad de la mucosa }(<5 \mathrm{~mm}) \text {, de color } \\
\text { blanco o amarillo, de bordes planos }\end{array}$ \\
\hline & $\begin{array}{l}\text { Úlcera superficial } \\
2 \text { puntos }\end{array}$ & $\begin{array}{l}\text { Defectos de continuidad de la mucosa }>5 \mathrm{~mm} \text {, correspondientes a } \\
\text { pequeñas úlceras cubiertas por fibrina }\end{array}$ \\
\hline & $\begin{array}{l}\text { Úlcera profunda } \\
3 \text { puntos }\end{array}$ & $\begin{array}{l}\text { Defectos excavados de continuidad de la mucosa, correspondientes a } \\
\text { úlceras más profundas y de bordes levemente elevados }\end{array}$ \\
\hline
\end{tabular}

Esta escala busca etapificar la gravedad endoscópica de la colitis ulcerosa, se informa el segmento más comprometido y su puntaje varía entre 0 y 8 puntos. Se considera remisión endoscópica un puntaje de 0 o 1 punto (con 0 puntos tanto en sangrado como en erosiones y úlceras) $)^{44-47}$.

A

\begin{tabular}{|c|c|c|c|c|c|c|}
\hline & Recto & \begin{tabular}{|l} 
Sigmoides \\
Colon Izq.
\end{tabular} & \begin{tabular}{|l|} 
Colon \\
Transverso \\
\end{tabular} & $\begin{array}{l}\text { Colon } \\
\text { Derecho }\end{array}$ & íleon & Total \\
\hline $\begin{array}{l}\text { Úlcera profunda } \\
\text { (si: } 12 \text { ptos/no: 0 ptos) }\end{array}$ & 0 & 0 & 0 & 12 & 0 & 12 \\
\hline $\begin{array}{l}\text { Úlcera superficial } \\
\text { (si: } 6 \text { ptos/no: } 0 \text { ptos) }\end{array}$ & 0 & 0 & 6 & 6 & 0 & 12 \\
\hline $\begin{array}{l}\text { Superficie } \\
\text { comprometida, en cm }\end{array}$ & 0 & 2 & 6 & 8 & 0 & 16 \\
\hline $\begin{array}{l}\text { Superficie ulcerada, en } \\
\mathrm{cm}\end{array}$ & 0 & 0 & 1.5 & 6 & 0 & 7.5 \\
\hline \multirow{2}{*}{\multicolumn{6}{|c|}{$\begin{array}{r}\text { Total } \\
\text { Segmentos examinados }\end{array}$}} & 47.5 \\
\hline & & & & & & 5 \\
\hline \multicolumn{6}{|c|}{ Total /Segmentos examinados (A) } & 9.5 \\
\hline \multicolumn{6}{|c|}{ Agregar 3 puntos si estenosis ulcerada (B) } & 0 \\
\hline \multicolumn{6}{|c|}{ Agregar 3 puntos si estenosis no ulcerada (C) } & 3 \\
\hline \multicolumn{6}{|c|}{ CDEIS (total $A+B+C$ ) } & 12.5 \\
\hline
\end{tabular}

B

\begin{tabular}{|c|c|c|c|c|c|c|}
\hline & Recto & $\begin{array}{l}\text { Sigmoides } \\
\text { Colon Izq. }\end{array}$ & $\begin{array}{l}\text { Colon } \\
\text { Transverso }\end{array}$ & $\begin{array}{l}\text { Colon } \\
\text { Derecho }\end{array}$ & íleon & Total \\
\hline $\begin{array}{l}\text { Presencia y tamaño de } \\
\text { úlceras }(0-3)\end{array}$ & 0 & 2 & 1 & 1 & 0 & 4 \\
\hline $\begin{array}{l}\text { Extensión superficie } \\
\text { ulcerada }(0-3)\end{array}$ & 0 & 1 & 1 & 1 & 0 & 3 \\
\hline $\begin{array}{l}\text { Extensión superficie } \\
\text { afectada }(0-3)\end{array}$ & 0 & 2 & 1 & 1 & 0 & 4 \\
\hline $\begin{array}{l}\text { Presencia y tipo de } \\
\text { estenosis (0-3) }\end{array}$ & 0 & 3 & 0 & 0 & 0 & 3 \\
\hline \multicolumn{6}{|c|}{ SES-CD (Total) } & 14 \\
\hline
\end{tabular}

Figura 6. Escalas de gravedad endoscópica para enfermedad de Crohn. A: Ejemplo de cálculo del CDEIS (Crohn's disease endoscopic index of severity). De acuerdo a esta escala, la enfermedad se considera inactiva con 0-2 puntos; actividad leve con 3-9 puntos; moderada, 9-12 puntos; grave > 12 puntos. B: Ejemplo de cálculo del SES-CD (Simple Endoscopic Score for Crohn's Disease). De acuerdo a esta escala, la enfermedad se considera inactiva con 0-2 puntos; actividad leve con 3-6 puntos; moderada, 7-15 puntos; grave $>15$ puntos $^{48-50}$. 
Tabla 6. Índice endoscópico de Rutgeerts

\begin{tabular}{|lc|}
\hline Hallazgos endoscópicos a nivel de la anastomosis ileo-cólica & Puntaje \\
\hline Sin lesiones (mucosa normal) & i0 \\
Menos de 5 lesiones aftoideas & $i 1$ \\
$\begin{array}{l}\text { Más de } 5 \text { lesiones aftoideas rodeadas por mucosa normal o lesiones confinadas a la anastomosis } \\
\text { ( }<1 \mathrm{~cm} \text { de longitud) }\end{array}$ & $\mathrm{i} 2$ \\
\hline Ileitis aftoidea difusa con mucosa difusamente inflamada & $\mathrm{i3}$ \\
\hline Inflamación difusa con úlceras, nódulos y/o estenosis & $\mathrm{i} 4$ \\
\hline
\end{tabular}

Se considera reactivación endoscópica un puntaje $\geq$ i2. En nuestra legislación, dada la mayor probabilidad de evolucionar con reactivación clínica de la enfermedad, se considera reactivación endoscópica significativa un puntaje $\geq$ i3 ${ }^{51-53}$.

\section{Conclusiones}

La endoscopia digestiva alta y la colonoscopia son exámenes cualitativos, por lo tanto, la descripción estandarizada de los hallazgos facilita la redacción y la interpretación de los informes. Las clasificaciones y escalas facilitan este proceso. Lamentablemente, sin un proceso de validación apropiado no se puede asegurar que una escala cuantifica correctamente lo que dice medir, que su variación sea consistente con la mejoría o deterioro de la enfermedad que intenta cuantificar y que sus mediciones sean reproducibles. Aunque no todas las escalas y clasificaciones detalladas en este artículo han sido totalmente validadas en el sentido metodológico estricto, la mayoría tienen el respaldo suficiente para ser usadas en los informes endoscópicos o, al menos, son útiles para facilitar la descripción de los hallazgos.

Esta revisión se ha centrado en clasificaciones endoscópicas tradicionales que utilizan solo luz blanca, sin magnificación ni tinción. El desarrollo tecnológico actual nos permite visualizar en detalle, tanto el patrón glandular, como las estructuras vasculares submucosas. Este nuevo campo de observación se ha acompañado de nuevas clasificaciones, las que deben franquear el mismo proceso metodológico para demostrar su validez. Describir y analizar estas clasificaciones se escapan del objetivo de esta revisión y serán discutidas en un artículo posterior.

En suma, conocer y divulgar estas herramientas permite utilizar un lenguaje común, tanto para los endoscopistas que ejecutan estos procedimientos, como para los médicos especialistas y no especialistas que deben interpretar estos resultados. Una construcción e interpretación apropiada de los reportes endoscópicos se traduce en menos errores diagnósticos, lo que trae beneficios directos para los pacientes.

\section{Referencias}

1. Hyland ME. The validity of health assessments: resolving some recent differences. J Clin Epidemiol 1993; 46 (9): 1019-23.

2. Bartlett SJ, Barbic SP, Bykerk VP, Choy EH, Alten R, Christensen R, et al. Content and Construct Validity, Reliability, and Responsiveness of the Rheumatoid Arthritis Flare Questionnaire: OMERACT 2016 Workshop Report. J Rheumatol 2017; 44 (10): 1536-43.

3. Charalambous C, Koulori A, Vasilopoulos A, Roupa Z. Evaluation of the Validity and Reliability of the Waterlow Pressure Ulcer Risk Assessment Scale. Med Arch (Sarajevo, Bosnia Herzegovina) 2018; 72 (2): 141-4.

4. Kahrilas PJ. Clinical practice. Gastroesophageal reflux disease. N Engl J Med 2008; 359 (16): 1700-7.

5. Zagari RM, Fuccio L, Wallander M-A, Johansson S, Fiocca R, Casanova S, et al. Gastro-oesophageal reflux symptoms, oesophagitis and Barrett's oesophagus in the general population: the Loiano-Monghidoro study. Gut 2008; 57 (10): 1354-9.

6. Frazzoni M, De Micheli E, Savarino V. Different patterns of oesophageal acid exposure distinguish complicated reflux disease from either erosive reflux oesophagitis or non-erosive reflux disease. Aliment Pharmacol Ther 2003; 18 (11-12): 1091-8.

7. Armstrong D, Bennett JR, Blum AL, Dent J, De Dombal FT, Galmiche JP, et al. The endoscopic assessment of esophagitis: a progress report on observer agreement. Gastroenterology 1996; 111 (1): 85-92.

8. Lundell LR, Dent J, Bennett JR, Blum AL, Armstrong D, Galmiche JP, et al. Endoscopic assessment of oesophagitis: clinical and functional correlates and further 
validation of the Los Angeles classification. Gut 1999; 45 (2): 172-80.

9. Wong R-KM, Yeoh K-G, Gwee K-A, Tay H-W, Ho K-Y. Validation of structured scoring using the LA classification for esophagitis and endoscopically suspected Barrett's esophagus in a tertiary Asian endoscopy center. J Gastroenterol Hepatol 2009; 24 (1): 103-6.

10. Gyawali CP, Kahrilas PJ, Savarino E, Zerbib F, Mion F, Smout AJPM, et al. Modern diagnosis of GERD: the Lyon Consensus. Gut 2018; 67 (7): 1351-62.

11. Savary M. Les hernies hiatales non compliquées. Endoscopic. Maladie peptique oesophagienne et gastrins herniaires. Med Hyg 1968; 26: 789.

12. Hill LD, Kozarek RA, Kraemer SJ, Aye RW, Mercer CD, Low DE, et al. The gastroesophageal flap valve: in vitro and in vivo observations. Gastrointest Endosc 1996; 44 (5): 541-7.

13. Contractor QQ, Akhtar SS, Contractor TQ. Endoscopic esophagitis and gastroesophageal flap valve. J Clin Gastroenterol 1999; 28 (3): 233-7.

14. Sharma P, Dent J, Armstrong D, Bergman JJGHM, Gossner L, Hoshihara Y, et al. The Development and Validation of an Endoscopic Grading System for Barrett's Esophagus: The Prague C \&amp; M Criteria. Gastroenterology 2006; 131 (5): 1392-9.

15. Lee YC, Cook MB, Bhatia S, Chow WH, El-Omar EM, Goto $\mathrm{H}$, et al. Interobserver reliability in the endoscopic diagnosis and grading of Barrett's esophagus: an Asian multinational study. Endoscopy 2010; 42 (9): 699-704.

16. Alvarez Herrero L, Curvers W, van Vilsteren F, Wolfsen $\mathrm{H}$, Ragunath K, Wong Kee Song L-M, et al. Validation of the Prague C\&amp;M classification of Barrett's esophagus in clinical practice. Endoscopy 2013; 45 (11): 876-82.

17. Vahabzadeh $B$, Seetharam AB, Cook MB, Wani S, Rastogi A, Bansal A, et al. Validation of the Prague C \&amp; $M$ criteria for the endoscopic grading of Barrett's esophagus by gastroenterology trainees: a multicenter study. Gastrointest Endosc 2012; 75 (2): 236-41.

18. Sharma P, Morales TG, Sampliner RE. Short segment Barrett's esophagus-the need for standardization of the definition and of endoscopic criteria. Am J Gastroenterol 1998; 93 (7): 1033-6.

19. Thota PN, Vennalaganti P, Vennelaganti S, Young P, Gaddam S, Gupta N, et al. Low Risk of High-Grade Dysplasia or Esophageal Adenocarcinoma Among Patients With Barrett's Esophagus Less Than $1 \mathrm{~cm}$ (Irregular Z Line) Within 5 Years of Index Endoscopy. Gastroenterology 2017; 152 (5): 987-92.

20. Paquet KJ. Prophylactic Endoscopic Sclerosing Treatment of the Esophageal Wall in Varices - A Prospective
Controlled Randomized Trial. Endoscopy 1982; 14 (01): 4-5.

21. Beppu K, Inokuchi K, Koyanagi N, Nakayama S, Sakata $\mathrm{H}$, Kitano S, et al. Prediction of variceal hemorrhage by esophageal endoscopy. Gastrointest Endosc 1981; 27 (4): 213-8.

22. North Italian Endoscopic Club for the Study and Treatment of Esophageal Varices. Prediction of the first variceal hemorrhage in patients with cirrhosis of the liver and esophageal varices. A prospective multicenter study. N Engl J Med 1988; 319 (15): 983-9.

23. Calès $\mathrm{P}$, Zabotto B, Meskens $\mathrm{C}$, Caucanas JP, Vinel JP, Desmorat $\mathrm{H}$, et al. Gastroesophageal endoscopic features in cirrhosis. Observer variability, interassociations, and relationship to hepatic dysfunction. Gastroenterology 1990; 98 (1): 156-62.

24. de Franchis R, Baveno VI Faculty. Expanding consensus in portal hypertension: Report of the Baveno VI Consensus Workshop: Stratifying risk and individualizing care for portal hypertension. J Hepatol 2015; 63 (3): 743-52.

25. Sarin SK, Kumar A. Gastric varices: profile, classification, and management. Am J Gastroenterol 1989; 84 (10): 1244-9.

26. Sarin SK, Lahoti D, Saxena SP, Murthy NS, Makwana UK. Prevalence, classification and natural history of gastric varices: a long-term follow-up study in 568 portal hypertension patients. Hepatology 1992; 16 (6): 1343-9.

27. Forrest JA, Finlayson ND, Shearman DJ. Endoscopy in gastrointestinal bleeding. Lancet (London, England) 1974; 2 (7877): 394-7.

28. de Groot NL, van Oijen MGH, Kessels K, Hemmink M, Weusten BLAM, Timmer R, et al. Reassessment of the predictive value of the Forrest classification for peptic ulcer rebleeding and mortality: can classification be simplified? Endoscopy 2014; 46 (1): 46-52.

29. Lanas A, Dumonceau J-M, Hunt RH, Fujishiro M, Scheiman JM, Gralnek IM, et al. Non-variceal upper gastrointestinal bleeding. Nat Rev Dis Prim 2018; 4: 18020.

30. Aiko T, Sasako M. The new Japanese Classification of Gastric Carcinoma: Points to be revised. Gastric Cancer 1998; 1 (1): 25-30.

31. The Paris endoscopic classification of superficial neoplastic lesions: esophagus, stomach, and colon: November 30 to December 1, 2002. Gastrointest Endosc 2003; 58 (6 Suppl): S3-43.

32. van Doorn SC, Hazewinkel Y, East JE, van Leerdam ME, Rastogi A, Pellisé M, et al. Polyp morphology: an interobserver evaluation for the Paris classification among international experts. Am J Gastroenterol 2015; 110 (1): $180-7$. 
33. Lai EJ, Calderwood AH, Doros G, Fix OK, Jacobson BC. The Boston bowel preparation scale: a valid and reliable instrument for colonoscopy-oriented research. Gastrointest Endosc 2009; 69 (3 Pt 2): 620-5.

34. Calderwood AH, Schroy PC, Lieberman DA, Logan JR, Zurfluh M, Jacobson BC. Boston Bowel Preparation Scale scores provide a standardized definition of adequate for describing bowel cleanliness. Gastrointest Endosc 2014; 80 (2): 269-76.

35. Parmar R, Martel M, Rostom A, Barkun AN. Validated Scales for Colon Cleansing: A Systematic Review. Am J Gastroenterol 2016; 111 (2): 197-204; quiz 205.

36. Satsangi J, Silverberg MS, Vermeire S, Colombel J-F. The Montreal classification of inflammatory bowel disease: controversies, consensus, and implications. Gut 2006; 55 (6): 749-53.

37. Silverberg MS, Satsangi J, Ahmad T, Arnott IDR, Bernstein CN, Brant SR, et al. Toward an integrated clinical, molecular and serological classification of inflammatory bowel disease: report of a Working Party of the 2005 Montreal World Congress of Gastroenterology. Can J Gastroenterol 2005; 19 Suppl A: 5A-36A.

38. Ungaro R, Colombel J-F, Lissoos T, Peyrin-Biroulet L. A Treat-to-Target Update in Ulcerative Colitis: A Systematic Review. Am J Gastroenterol 2019; 114 (6): 874-83.

39. Shah SC, Colombel JF, Sands BE, Narula N. Mucosal Healing Is Associated With Improved Long-term Outcomes of Patients With Ulcerative Colitis: A Systematic Review and Meta-analysis. Clin Gastroenterol Hepatol 2016; 14 (9): 1245-55.e8.

40. Shah SC, Colombel JF, Sands BE, Narula N. Systematic review with meta-analysis: Mucosal healing is associated with improved long-term outcomes in Crohn's disease. Aliment Pharmacol Ther 2016; 43 (3): 317-33.

41. Yzet C, Diouf M, Le Mouel J-P, Brazier F, Turpin J, Loreau J, et al. Complete Endoscopic Healing Associated With Better Outcomes Than Partial Endoscopic Healing in Patients With Crohn's Disease. Clin Gastroenterol Hepatol 2019.

42. Schroeder KW, Tremaine WJ, Ilstrup DM. Coated Oral 5-Aminosalicylic Acid Therapy for Mildly to Moderately Active Ulcerative Colitis. N Engl J Med 1987; 317 (26): 1625-9.

43. Ministerio de Salud Chile. Protocolo Ley Ricarte Soto: Tratamiento con Golimumab o Adalimumab para personas con Colitis Ulcerosa moderada e Infliximab en personas con Colitis Ulcerosa grave, refractaria al tratamiento de primera línea. [Internet]. 2019 [citado el 7 de junio de 2020]. Available from: https://www.minsal. cl/wp-content/uploads/2019/07/20.-10072019-Protoco-

\section{lo-CUlcerosa_FINAL.pdf}

44. Vuitton L, Peyrin-Biroulet L, Colombel JF, Pariente B, Pineton de Chambrun G, Walsh AJ, et al. Defining endoscopic response and remission in ulcerative colitis clinical trials: an international consensus. Aliment Pharmacol Ther 2017; 45 (6): 801-13.

45. Travis SPL, Schnell D, Krzeski P, Abreu MT, Altman DG, Colombel J-F, et al. Developing an instrument to assess the endoscopic severity of ulcerative colitis: the Ulcerative Colitis Endoscopic Index of Severity (UCEIS). Gut 2012; 61 (4): 535-42.

46. Samuel S, Bruining DH, Loftus E V, Thia KT, Schroeder KW, Tremaine WJ, et al. Validation of the ulcerative colitis colonoscopic index of severity and its correlation with disease activity measures. Clin Gastroenterol Hepatol 2013; 11 (1): 49-54.e1.

47. Mohammed Vashist N, Samaan M, Mosli MH, Parker CE, MacDonald JK, Nelson SA, et al. Endoscopic scoring indices for evaluation of disease activity in ulcerative colitis. Cochrane Database Syst Rev 2018; 1: CD011450.

48. Mary JY, Modigliani R. Development and validation of an endoscopic index of the severity for Crohn's disease: a prospective multicentre study. Groupe d'Etudes Thérapeutiques des Affections Inflammatoires du Tube Digestif (GETAID). Gut 1989; 30 (7): 983-9.

49. Daperno M, D'Haens G, Van Assche G, Baert F, Bulois P, Maunoury V, et al. Development and validation of a new, simplified endoscopic activity score for Crohn's disease: the SES-CD. Gastrointest Endosc 2004; 60 (4): 505-12.

50. Khanna R, Nelson SA, Feagan BG, D'Haens G, Sandborn WJ, Zou GY, et al. Endoscopic scoring indices for evaluation of disease activity in Crohn's disease. Cochrane database Syst Rev 2016; 8: CD010642.

51. Rutgeerts P, Geboes K, Vantrappen G, Beyls J, Kerremans R, Hiele M. Predictability of the postoperative course of Crohn's disease. Gastroenterology 1990; 99 (4): 956-63.

52. De Cruz P, Kamm MA, Hamilton AL, Ritchie KJ, Krejany EO, Gorelik A, et al. Crohn's disease management after intestinal resection: a randomised trial. Lancet (London, England) 2015; 385 (9976): 1406-17.

53. Ministerio de Salud Chile. Protocolo Ley Ricarte Soto: Tratamiento con Infliximab o Adalimumab para personas con Enfermedad de Crohn grave Refractaria a Tratamiento Habitual [Internet]. 2019 [citado el 7 de junio de 2020]. Available from: https://www.minsal. cl/wp-content/uploads/2019/07/6.-10072019-Protocolo-CROHN-II_FINAL.pdf 